\title{
Ethanol extract of gardenia fruit alleviates renal interstitial fibrosis induced by unilateral ureteral obstruction in rats
}

\author{
XIAOBO LI ${ }^{1,2}$, MIN MA $^{1}$, XIANGGUI ZHANG ${ }^{2}$, LIANG DENG $^{3}$, YARONG WANG $^{4}$, \\ ZHUANG BIAN ${ }^{5}$, SHINING CAI ${ }^{4}$, BANGYA PENG $^{4}$, JIANGQUAN YANG $^{4}$ and YANG CHEN ${ }^{4}$ \\ ${ }^{1}$ College of Traditional Chinese Medicine, Jinan University, Guangzhou, Guangdong 510632; \\ ${ }^{2}$ Department of Diagnostics, Zhuhai Campus of Zunyi Medical University, Zhuhai, Guangdong 519041; \\ ${ }^{3}$ School of Pharmaceutical Science and Yunnan Key Laboratory of Pharmacology for Natural Products, \\ Kunming Medical University, Kunming, Yunnan 650500; ${ }^{4}$ Department of Pharmaceutical Sciences, \\ Zhuhai Campus of Zunyi Medical University, Zhuhai, Guangdong 519041; ${ }^{5}$ Department of Gastroenterology, \\ The Fifth Affiliated Hospital of Sun Yat-sen University, Zhuhai, Guangdong 519000, P.R. China
}

Received May 10, 2016; Accepted April 10, 2017

DOI: 10.3892/etm.2017.4662

\begin{abstract}
Gardenia fruit has been used in traditional Chinese medicine for thousands of years. A previous study by the present authors indicated that the ethanol extract of gardenia fruits (EEG) primarily contains eight constituents. In the present study, the potential effects of EEG on unilateral ureteral obstruction (UUO)-induced renal interstitial fibrosis were observed in rats. A total of 30 rats were randomly divided into three groups ( $\mathrm{n}=10$ each): Sham group, UUO group, and EEG group, which were administered with EEG (200 mg/kg/day) or the same volume of distilled water as a vehicle. UUO were established by ligating left ureter at two points and cut between the ligatures. All rats were sacrificed at 14 days after UUO operation. the present results demonstrated that EEG significantly elevated the expressions of vascular endothelial growth factor and E-cadherin induced by UUO (both $\mathrm{P}<0.05$ ), and reduced levels of hypoxia-inducible factor- $1 \alpha$, transforming growth factor- $\beta 1$, connective tissue growth factor and $\alpha$-smooth muscle actin (all $\mathrm{P}<0.05$ ). The present findings suggest that EEG is a potential novel renoprotective compound for renal fibrosis through inhibiting epithelial-to-mesenchymal transition.
\end{abstract}

Correspondence to: Professor Yang Chen, Department of Pharmaceutical Sciences, Zhuhai Campus of Zunyi Medical University, 368 Jinwan Road, Zhuhai, Guangdong 519041, P.R. China E-mail: chenyang197732@hotmail.com

Key words: ethanol extract of gardenia fruits, hypoxia-inducible factor-1 $\alpha$, vascular endothelial growth factor, transforming growth factor- $\beta_{1}$, connective tissue growth factor

\section{Introduction}

Chronic kidney disease (CKD) is a major healthcare burden and the $18^{\text {th }}$ leading cause of disease-related mortality worldwide, with an estimated prevalence of $8-16 \%$ worldwide $(1,2)$. Renal interstitial fibrosis (RIF) is the major pathological basis for many types of CKD, and the inhibition of RIF has been considered as an effective therapeutic strategy to protect the kidney (3). RIF is generally characterized by an excessive accumulation and deposition of extracellular matrix (ECM) that progressively leads to the degradation of functional nephrons. This ECM is predominantly produced by $\alpha$-smooth muscle actin ( $\alpha$-SMA)-expressing myofibroblasts, which may originate from renal epithelial tubules through epithelial-to-mesenchymal transition (EMT) (4). EMT is able to induce a downregulation of E-cadherin in epithelial cells, which leads to a loss of adhesion and polarity in these cells (5). In the process of EMT, the expression of myofibroblast cell marker $\alpha$-SMA is increased in tubular epithelial cells, whereas epithelial marker E-cadherin expression is decreased (6).

Transforming growth factor- $\beta 1$ (TGF- $\beta 1$ ) has generally been considered as an essential factor in RIF (7) and it is recognized that TGF- $\beta 1$ and its downstream mediator, connective tissue growth factor (CTGF), are associated with most forms of CKD. EMT and fibrosis are promoted by the expression of TGF- $\beta 1$, whereas they are prevented by the inhibition of TGF- $\beta 1$ by various methods (8). CTGF is also known to be an important matrix-cellular regulatory factor, which participates in angiogenesis, and appears to be a central mediator of EMT and fibrosis (9).

In addition, fibrosis is able to induce ischemia and hypoxia, and hypoxia-inducible factor- $1 \alpha(\mathrm{HIF}-1 \alpha)$ is a transcription factor associated with cellular responses to hypoxia; recent reports have suggested that HIF-1 $\alpha$ has an important role in stimulating EMT in vivo $(10,11)$. Furthermore, vascular endothelial growth factor (VEGF) is a downstream factor of HIF-1 $\alpha$, which promotes endothelial cell proliferation, induces 
microvascular hyperpermeability and is associated with interstitial matrix remodeling $(12,13)$.

The fruit of Gardenia jasminoides Ellis (Rubiaceae) has been used in Traditional Chinese Medicine for the treatment of fever, jaundice, headache, edema, hypertension, and hepatic disorders. Gardenia extract contains multiple active chemical components, and a previous study by the present authors indicated that the ethanol extract of gardenia fruits (EEG) primarily contains eight constituents (14). Previous studies have indicated that these components have various pharmacological properties, including anti-inflammatory effects (15-17) and the ability to attenuate oxidative stress (18); notably, inflammation and oxidative stress are critical pathogenic factors in fibrogenesis. Gardenia has previously been reported to exert antifibrotic effects in liver fibrosis (19) However, to the best of our knowledge, there are currently no published studies regarding the antifibrotic effect of EEG in renal tissues.

Unilateral ureteral obstruction (UUO) is the most frequently used experimental model for the investigation of the mechanisms of RIF and for the evaluation of therapeutic methods to alleviate fibrosis. Therefore, the effects of EEG on UUO-induced RIF were studied in vivo. Furthermore, potential underlying mechanisms were also explored. The present results demonstrated that EEG was able to inhibit the induction of EMT by decreasing TGF- $\beta 1$, CTGF, HIF-1a, and increasing VEGF expression.

\section{Materials and methods}

Plant materials and reagents. The following antibodies were used for immunohistochemistry and western blotting: Rabbit polyclonal antibodies against HIF- $1 \alpha$, VEGF, TGF- $\beta 1$, CTGF, $\beta$-actin, goat anti rabbit IgG-Biotin (cat. nos: PB2045, BA0407, BA0290, BA0752, BA2305 and BA1003, respectively; all Wuhan Boster Biological Technology, Ltd., Wuhan, China), E-cadherin and $\alpha$-SMA (cat. nos: 20874 and 14395; Wuhan Sanying Biotechnology, Wuhan, China). An SABC kit (cat. no.: SA1022; Wuhan Boster Biological Technology, Ltd.), NAG ELISA kit (cat. no.: RA20482, Shanghai Qiaodu Biomart, Shanghai, China), bicinchoninic acid assay protein quantification kit and enhanced chemiluminescence plus kit (cat. nos: P0010, P0018; Beyotime Institute of Biotechnology, Haimen, China) were also purchased. A Masson Trichrome staining kit (cat. no.: DC0033; Beijing Leagene Biotechnology Co., Ltd. Beijing, China), bovine serum albumin (BSA; Sangon Biotech, Shanghai, China), radioimmunoprecipitation assay (RIPA) lysis buffer (Shanghai Solarbio Biotechnology Co., Ltd. Shanghai, China) and chloral hydrate (Shanghai Sinopharm group, Shanghai, China) were used in the present study. The dried gardenia fruits were purchased from Yuanchunlin Pharmacy (Zhuhai, China) in January 2014 and identified by Yang Chen. Voucher specimens were deposited at the Department of Pharmaceutical Sciences, Zunyi Medical University Zhuhai Campus (Zhuhai, China). Reference gardenoside, $6 \beta$-hydroxy geniposide, geniposidic acid, geniposide, crocin-1, crocin-2, crocin-3 and crocin-4, were isolated from EEG by high-speed counter-current chromatography in a previous study by the present authors (14). The structures of these compounds are displayed in Fig. 1.
Preparation of crude extracts and ultra performance liquid chromatography (UPLC) analysis. Chemical extraction and ultra performance liquid chromatography analysis were performed according to our previous study (14). Briefly, the dried gardenia fruits $(2 \mathrm{~kg})$ were ground to coarse powder and extracted with ethanol-water $(40 \%)$ via cold percolation ( $2 \times 2 \mathrm{l})$ at $25^{\circ} \mathrm{C}$ for $24 \mathrm{~h}$. The alcohol extract was concentrated by rotary evaporation, and the sample was stored at $4^{\circ} \mathrm{C}$ prior to animal experiments. The mobile phase was composed of acetonitrile (A) and pure water (B), that was programmed as follows: From 0-4.5 min, 10-18\% A; 4.5-6.0 min, linear gradient increase from 18-28\% A; 6.0-12.0 min, linear gradient increase from $28-38 \% \mathrm{~A} ; 12.0-15.0 \mathrm{~min}$, linear gradient increase from $38-50 \% \mathrm{~A} ; 15.0-20.0 \mathrm{~min}$, linear gradient increase from $50-10 \% \mathrm{~A}$. The flow rate was $0.3 \mathrm{ml} / \mathrm{min}$ and the detection wavelength was as follows: 0-7 $\mathrm{min}, 238 \mathrm{~nm}$; 7-20 min, $440 \mathrm{~nm}$.

Experimental groups and protocol. A total of 30 male 8-week-old Sprague-Dawley rats, weighing 180-220 g, were provided by the Animal Experimental Center of Guangdong Province (Guangzhou, China). Rats had free access to food and water at a temperature of $22 \pm 1^{\circ} \mathrm{C}$, a relative humidity of $55 \pm 2 \%$, and under a $12 \mathrm{~h} \mathrm{light/dark}$ cycle. The rats were randomly assigned into three groups $(n=10$ each): i) Sham-operation plus vehicle (sham group); ii) UUO plus vehicle (UUO group); and iii) UUO plus EEG (EEG group). Briefly, after anesthesia an intraperitoneal injection of $10 \%$ chloral hydrate $(300 \mathrm{mg} / \mathrm{kg})$, the left kidney and ureter were exposed via a flank incision. Then the left ureter was ligated at two points and cut between the ligatures. Sham group animals underwent an identical procedure without ligation. Rats were gavaged with EEG at a dose of $200 \mathrm{mg} / \mathrm{kg} /$ day in the EEG group, whereas rats in the sham and UUO groups were gavaged with the same volume of vehicle (distilled water) once daily. All rats were sacrificed 14 days after UUO surgery, the left kidneys were excised, and harvested for immunochemistry and western blotting. Blood and urine samples were harvested for biochemical analysis. The present methodology was approved by the Ethics Committee of Zunyi Medical University.

Physiological parameters. N-acetyl- $\beta$-d-glucosaminidase (NAG), which is a marker of renal tubular damage was measured in urine samples using an ELISA kit according to the manufacturer's protocol. Serum creatinine $(\mathrm{Cr})$ and blood urea nitrogen (BUN) levels were measured by a Roche automatic biochemical analysis.

Histopathologic examination. kidney tissues were fixed in $10 \%$ neutral formalin solution for $24 \mathrm{~h}$ at room temperature, embedded in paraffin, and sectioned into $4 \mu \mathrm{m}$ sections that were stained with hematoxylin-eosin (H\&E) and Masson's trichrome stains using a Masson Trichrome staining kit according to the manufacturer's protocol. A total of 10 non-consecutive high-power fields of each renal section were examined by light microscopy. The H\&E staining was used to provide a semi-quantitative score on the degree of tubulointerstitial lesions (20), and Masson staining was used to calculate the renal interstitial fibrosis area (21). 
Immunohistochemical analysis. Renal sections ( $4 \mu \mathrm{m})$ were cut from paraffin-embedded tissues. Sections were blocked with $5 \% \mathrm{BSA}$ at room temperature for $20 \mathrm{~min}$. Slides were incubated overnight at $4^{\circ} \mathrm{C}$ with the following primary antibodies: HIF-1 $\alpha$ (1:100), VEGF (1:100), E-cad (1:50), $\alpha$-SMA (1:50), TGF- $\beta 1$ (1:50) and CTGF (1:50). Following washing with PBS (3 times), slide sections were incubated at room temperature with the secondary antibody (1:100) for $20 \mathrm{~min}$. The immune complexes were then detected by the diaminobenzidine substrate. Finally, the brown reaction product was observed via light microscopy (magnification, x400). Furthermore, 10 non-consecutive visual fields were randomly selected in the renal section. The integrated optical density (IOD) total and mean density (IOD/area) of each visual field were determined using Image-Pro Plus software 6.0 (Media Cybernetics, Inc., Rockville, MD, USA).

Western blotting. In the kidney tissue-based assay, kidney tissue samples from each group were homogenized with lysis buffer plus $1 \mathrm{mM}$ PMSF and protease inhibitor cocktail. Proteins were treated with RIPA lysis buffer containing a cocktail of protease inhibitors $(2 \mu \mathrm{g} / \mathrm{ml}$ aprotinin, $1 \mathrm{mM}$ phenylmethyl sulfonylfuoride, and $10 \mu \mathrm{g} / \mathrm{ml}$ leupeptin). Lysates were incubated for $30 \mathrm{~min}$ at $4^{\circ} \mathrm{C}$ and centrifuged at $12,000 \mathrm{x} \mathrm{g}$ for $10 \mathrm{~min}$ at room temperature. Supernatant protein concentration was then determined using to a bicinchoninic acid protein quantification kit. Protein samples $(20 \mu \mathrm{g})$ were separated by $10 \%$ SDS-PAGE and transferred to a PVDF membrane using a wet transferring method. The membranes were blocked with $5 \% \mathrm{BSA}$ for $1 \mathrm{~h}$ at $4^{\circ} \mathrm{C}$ and incubated overnight at $4^{\circ} \mathrm{C}$ with primary antibodies against HIF-1 $\alpha$ (1:100), VEGF (1:100), E-cad (1:100), $\alpha$-SMA (1:100), TGF- $\beta 1$ (1:100), CTGF (1:100) and $\beta$-actin proteins (1:100). Membranes were subsequently incubated for $2 \mathrm{~h}$ at room temperature with horseradish peroxidase-conjugated antibodies (anti-rabbit $\mathrm{IgG} ; 1: 1,000$ ). Protein bands were visualized using an electrochemiluminescence kit and densitometric analysis of the western blot results was performed with Image J version 1.48 software (National Institutes of Health, Bethesda, MD, USA).

Statistical analysis. Experimental data were analyzed using SPSS 13.0 (SPSS, Inc., Chicago, IL, USA) and all data are presented as the mean \pm standard deviation. Student's t-test or one-way analysis of variance was used in statistical analysis of the data. $\mathrm{P}<0.05$ was considered to indicate a statistically significant difference.

\section{Results}

UPLC analysis of gardenia extract. Under the chromatographic conditions used in the present study, eight compounds were well-resolved from EEG with baseline separation, namely gardenoside, $6 \beta$-hydroxy geniposide, geniposidic acid, geniposide, crocin-1, crocin-2, crocin-3 and crocin-4 (Fig. 1). Identification of the compounds was confirmed by direct comparison of UV spectra and the retention time of each analyte with those obtained from the references. UPLC analysis of gardenia alcoholic extract indicates that iridoid glycosides and crocetin derivatives are the primary components of this herb. In addition, gardenoside, $6 \beta$-hydroxy
Table I. Physiological parameters of renal interstitial fibrosis model rats.

\begin{tabular}{lcccc}
\hline Group & $\begin{array}{c}\text { Rats } \\
(\mathrm{n})\end{array}$ & $\begin{array}{c}\text { Scr } \\
(\mu \mathrm{mol} / \mathrm{l})\end{array}$ & $\begin{array}{c}\mathrm{BUN} \\
(\mathrm{mmol} / \mathrm{l})\end{array}$ & $\begin{array}{c}\mathrm{NAG} \\
(\mathrm{U} / \mathrm{l})\end{array}$ \\
\hline Sham & 10 & $38.96 \pm 7.86$ & $6.09 \pm 0.97$ & $9.10 \pm 3.55$ \\
UUO & 10 & $42.41 \pm 4.64$ & $8.48 \pm 0.99$ & $27.12 \pm 9.89^{\mathrm{a}}$ \\
EEG & 10 & $40.25 \pm 4.01$ & $6.93 \pm 0.55$ & $12.35 \pm 4.76^{\mathrm{b}}$ \\
\hline
\end{tabular}

${ }^{\mathrm{a}} \mathrm{P}<0.05$ vs. sham group; ${ }^{\mathrm{b}} \mathrm{P}<0.05$ vs. UUO group. Scr, serum creatinine; BUN, blood urea nitrogen; NAG, N-acetyl- $\beta$-d-glucosaminidase; UUO, unilateral ureteral obstruction; EEG, ethanol extract of gardenia fruits.
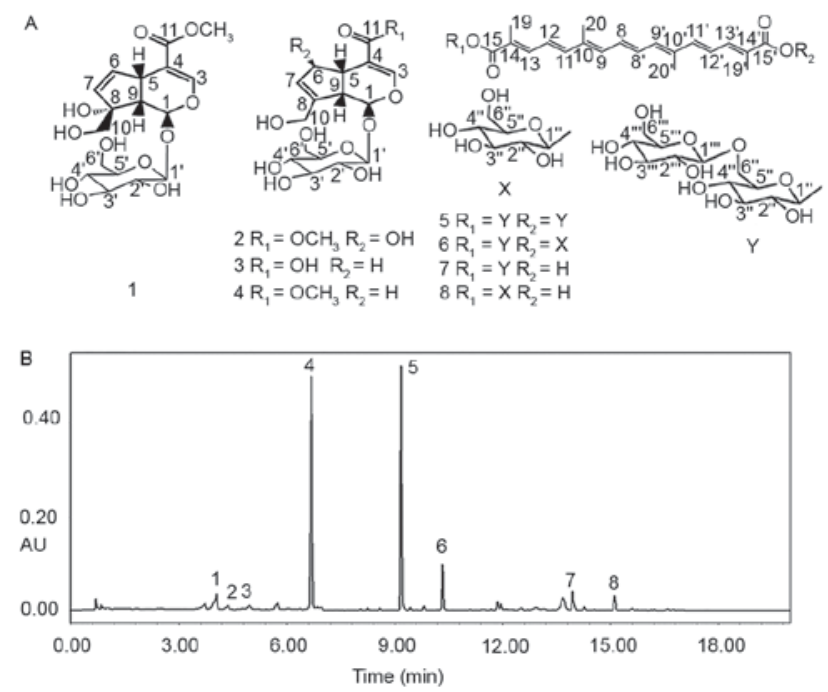

Figure 1. Structures and UPLC chromatogram of EEG. (A) Structures of compounds 1-8. (B) UPLC chromatogram of EEG. The detection wavelength was as follows: $0-7 \mathrm{~min}, 238 \mathrm{~nm} ; 7-20 \mathrm{~min}, 440 \mathrm{~nm}$. UPLC, ultra performance liquid chromatography; EEG, ethanol extract of gardenia fruits; 1, gardenoside; 2, 6ß-hydroxy geniposide; 3 , geniposidic acid; 4, geniposide; 5 , crocin- $1 ; 6$, crocin- $2 ; 7$, crocin $-3 ; 8$, crocin- 4 .

geniposide, geniposidic acid, geniposide, crocin-1, crocin-2, crocin-3 and crocin-4 were determined to account for 1.721 , $0.384,0.362,26.041,9.841,1.365,0.771$ and $0.785 \mathrm{~g} / 100 \mathrm{~g}$ ethanol extract of gardenia, respectively.

Physiological parameters. As presented in Table I, Compared with the sham group, NAG was significantly increased in the UUO group, whereas this was significantly decreased in the EEG group (both $\mathrm{P}<0.05$ ). No significant differences were observed in Scr and BUN levels among the three groups.

EEG ameliorates UUO-induced renal histologic changes. $\mathrm{H} \& \mathrm{E}$ staining (Fig. 2) demonstrated that kidney histology was normal in the sham group (Fig. 2A), whereas rats in the UUO group developed severe tubulointerstitial damage at 14 days following UUO operation (Fig. 2B). However, the EEG group exhibited an improved histological appearance, with attenuated inflammatory cellular infiltration, and reduced tubular expansion and atrophy (Fig. 2C). Significantly decreased the 

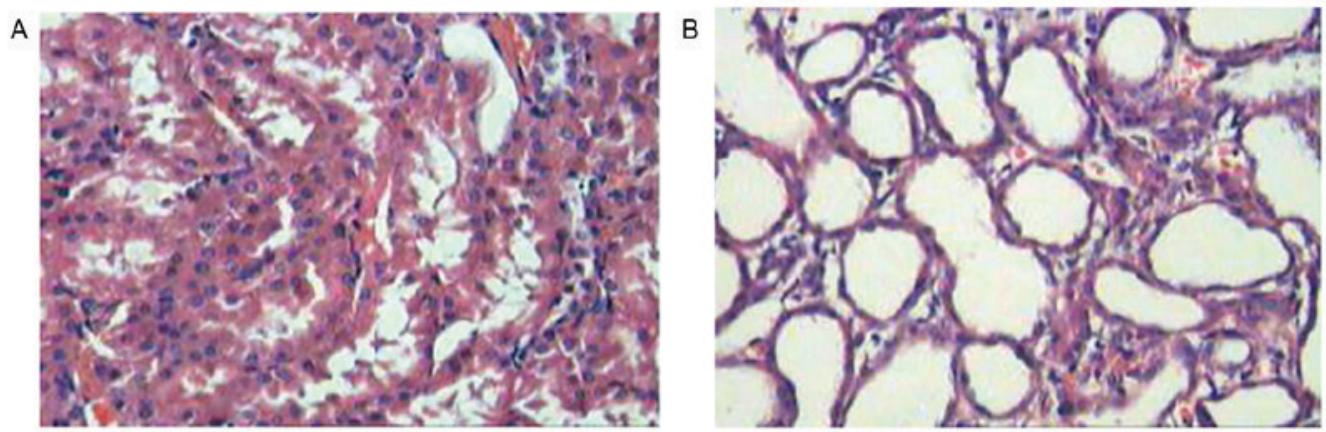

C

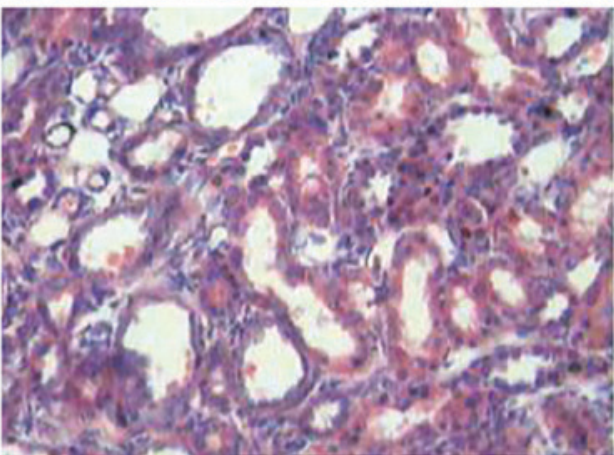

D

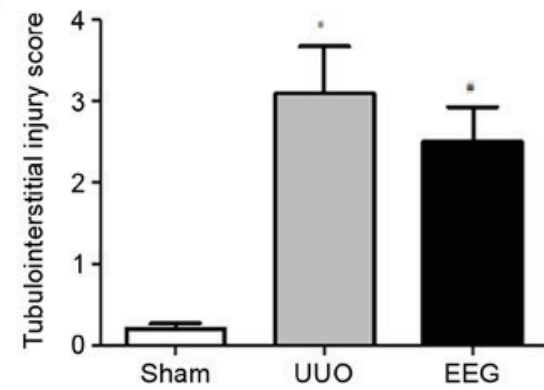

$\mathrm{E}$
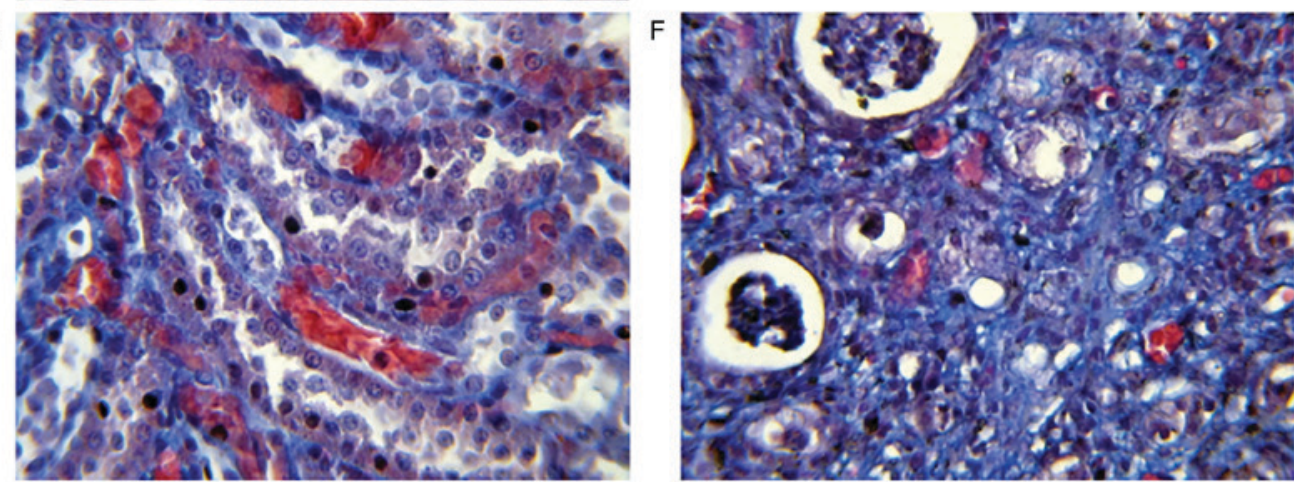

G

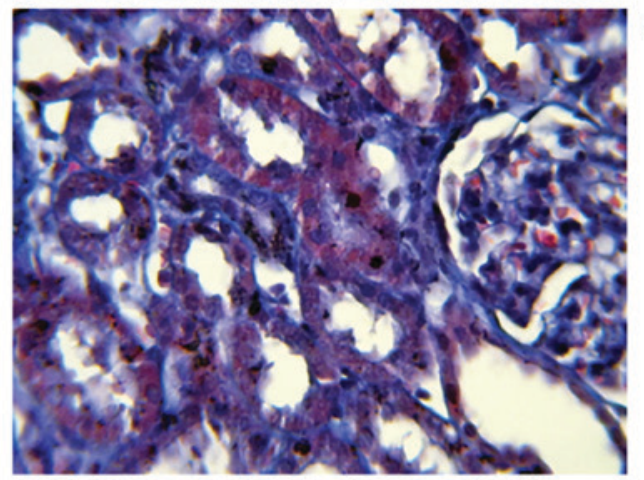

$\mathrm{H}$

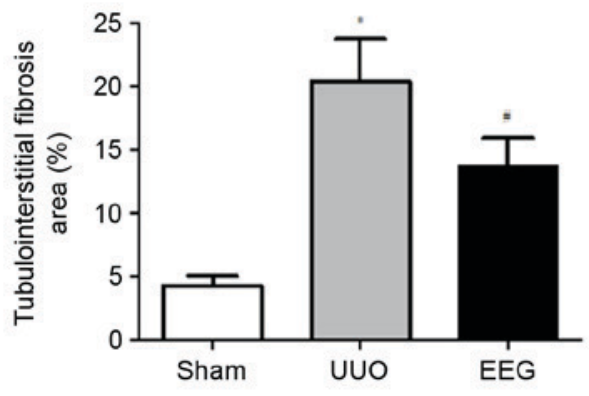

Figure 2. Morphological analysis of renal histology. Hematoxylin-eosin staining was performed to evaluate tubulointerstitial injury (A-D) and Masson staining was performed to evaluate tubulointerstitial fibrosis (E-H). (A and E) No marked histological abnormalities were observed in the sham group. (B and F) Renal fibrosis was clearly visible in the UUO group. (C and G) EEG markedly decreased tubulointerstitial injury and inflammatory cells infiltration. (D) Statistical analyses of tubulointerstitial injury scores. (H) Statistical analyses of relative percentages of tubulointerstitial fibrosis. Images were captured at a magnification of $\mathrm{x} 400$. Data are presented as the mean \pm standard deviation $(\mathrm{n}=10)$. ${ }^{*} \mathrm{P}<0.05$ vs. sham group; $\mathrm{P}<0.05$ vs. UUO group. UUO, unilateral ureteral obstruction; EEG, ethanol extract of gardenia fruits.

tubulointerstitial injury scores of obstructed rats $(\mathrm{P}<0.05$; Fig. 2D).

Effect of EEG on UUO-induced RIF. Masson staining indicated marked tubulointerstitial fibrosis in UUO group (Fig. 2F). Compared with the sham group (Fig. 2E), the percentage area of the fibrous area in the UUO group was significantly higher
$(\mathrm{P}<0.05)$, whereas this was significantly ameliorated by EEG treatment $(\mathrm{P}<0.05$; Fig. $2 \mathrm{G}$ and $\mathrm{H})$.

Immunostaining and western blot analysis of HIF-1 $\alpha$ and $V E G F$. Immunostaining and western blot analysis of HIF-1 $\alpha$ and VEGF are presented in Fig. 3. Compared with the sham group, HIF-1 $\alpha$ expression was markedly increased in fibrotic 
A

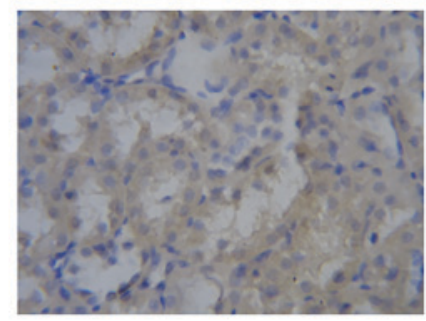

D

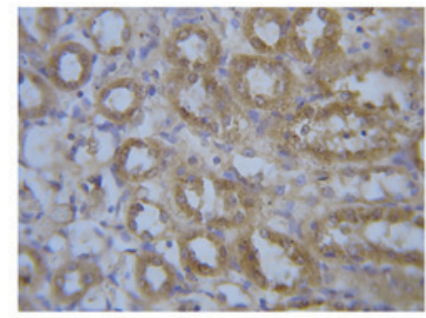

G

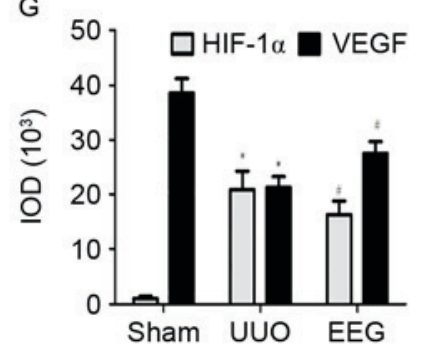

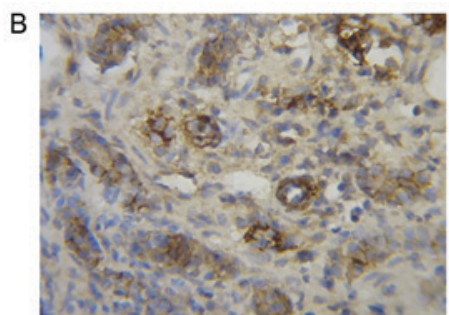

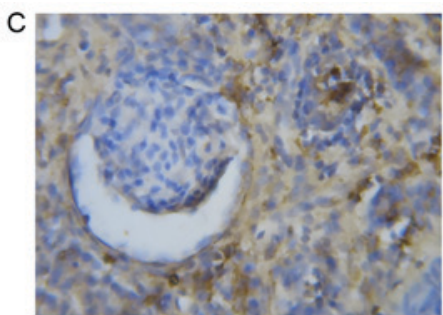

$\mathrm{E}$
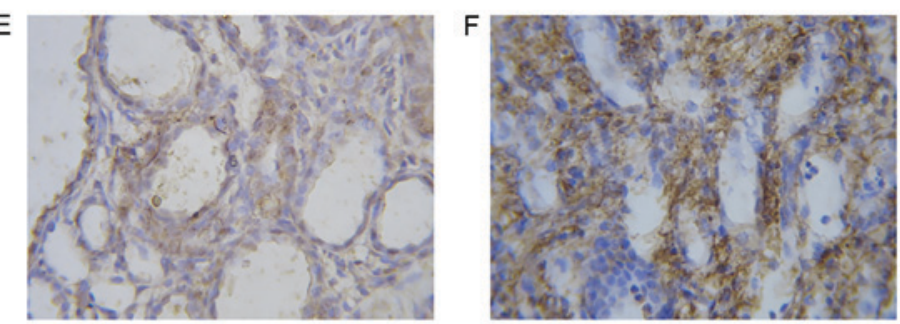

I

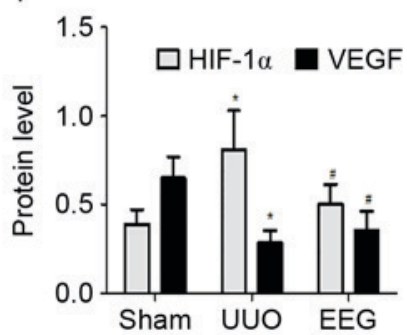

Figure 3. Immunostaining and western blot analysis of HIF-1 $\alpha$ and VEGF. (A) HIF-1 $\alpha$ staining was weak in the sham group. (B) HIF-1 $\alpha$ staining was markedly higher in the UUO group. (C) HIF-1 $\alpha$ expression was inhibited in the EEG group. (D) VEGF was most markedly expressed in the sham group. (E) VEGF staining was markedly reduced in the UUO group. (F) VEGF staining was markedly increased in the EEG group. (G) The total IOD of HIF-1 $\alpha$ and VEGF in each group. (H and I) Western blot analysis of HIF-1 $\alpha$ and VEGF demonstrated similar trends as the immunohistochemistry findings. Images were captured at a magnification of $\mathrm{x} 400$. Data are presented as the mean \pm standard deviation $(\mathrm{n}=10)$. ${ }^{*} \mathrm{P}<0.05$ vs. sham group; ${ }^{\sharp} \mathrm{P}<0.05$ vs. UUO group. HIF-1 $\alpha$, hypoxia-inducible factor-1 $\alpha$; VEGF, vascular endothelial growth factor; UUO, unilateral ureteral obstruction; EEG, ethanol extract of gardenia fruits; IOD, integrated optical density.

areas and tubular epithelial cells in the UUO group (Fig. 3A and B). Compared with the UUO group, HIF-1 $\alpha$ staining was markedly decreased in tubules in the EEG group (Fig. 3C). Western blot analysis revealed that expression of HIF-1 $\alpha$ protein was significantly reduced in the EEG group compared with the UUO group $(\mathrm{P}<0.05$; Fig. $3 \mathrm{H}$ and $\mathrm{I})$, which was consistent with the immunohistochemistry ( $\mathrm{P}<0.05$; Fig. $3 \mathrm{G})$. This suggests that expression of HIF-1 $\alpha$ protein was reduced by EEG treatment.

VEGF was most prominent in tubular epithelial cells in the sham group (Fig. 3D). In the UUO group (Fig. 3E), the expression of VEGF was significantly decreased compared with the sham group $(\mathrm{P}<0.05)$, whereas the level of VEGF protein in the EEG group (Fig. 3E) was significantly increased compared with UUO group ( $\mathrm{P}<0.05$; Fig. $3 \mathrm{~F}$ and $\mathrm{G})$. The level of VEGF protein detected by western blotting exhibited trends similar to those observed in immunohistochemistry (Fig. $3 \mathrm{H}$ and I). These results suggest that the level of VEGF protein was enhanced in the EEG group.

Immunostaining and western blot analysis of TGF- $\beta 1$ and $C T G F$. Immunostaining and western blot analysis of TGF- $\beta 1$ and CTGF are presented in Fig. 4. As shown in Fig. 4A-C and G, there was a significant increase in TGF- $\beta 1$ expression in the interstitial cells and the tubular epithelial cells, and interstitial fibrotic regions of kidneys in the UUO group compared with the sham group $(\mathrm{P}<0.05)$; however, this increase was significantly inhibited by EEG $(\mathrm{P}<0.05)$. Similar observations were made in western blot analysis (Fig. $4 \mathrm{H}$ and I).

In the sham group, faint CTGF staining was detected in the interstitial cells (Fig. 4D) which was significantly increased in the UUO group $(\mathrm{P}<0.05$; Fig. $4 \mathrm{E}$ and $\mathrm{G})$. The expression of CTGF protein in the EEG group was significantly reduced compared with the UUO group (Fig. 4F). Similar observations were made in western blot analysis (Fig. $4 \mathrm{H}$ and I).

Immunostaining and western blot analysis of E-cadherin and $\alpha$-SMA. Immunostaining and western blot analysis of E-cadherin and $\alpha$-SMA are presented in Fig. 5. In the sham group, staining of E-cadherin was predominantly observed in the cytoplasmic membrane and the tubuloepithelial cells (Fig. 5A). There was a significant reduction in the level of E-cadherin in the UUO group compared with sham group $(\mathrm{P}<0.05$; Fig. 5B and $\mathrm{G})$; however, the level of E-cadherin in the EEG group was significantly increased compared with the UUO group ( $\mathrm{P}<0.05$; Fig. $5 \mathrm{C}$ and $\mathrm{G})$. Similar observations were made in western blot analysis (Fig. 5H and I).

In the sham group, faint $\alpha$-SMA staining was observed (Fig. 5D. There was a significant increase expression of $\alpha$-SMA in the UUO group compared with the sham group $(\mathrm{P}<0.05$; 
A

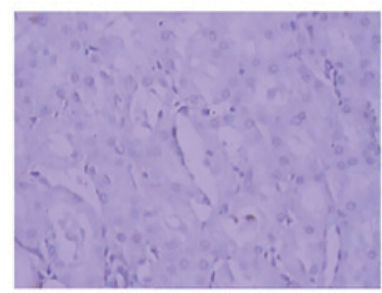

D

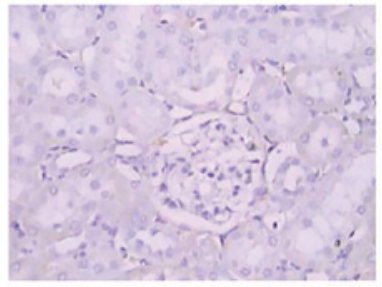

G

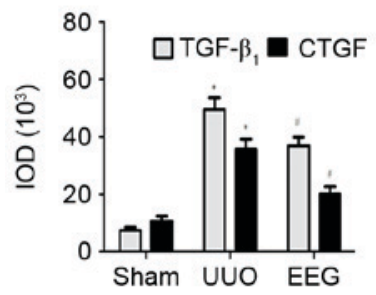

B
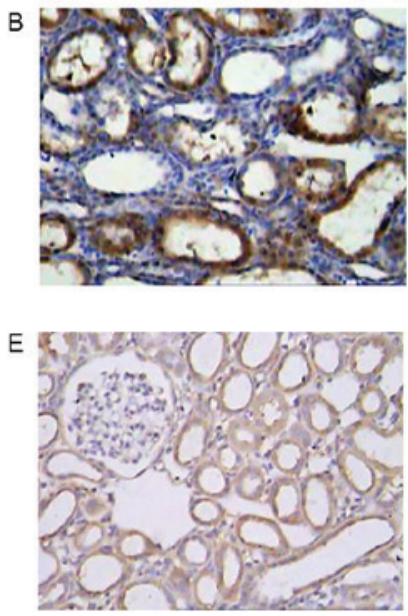

H

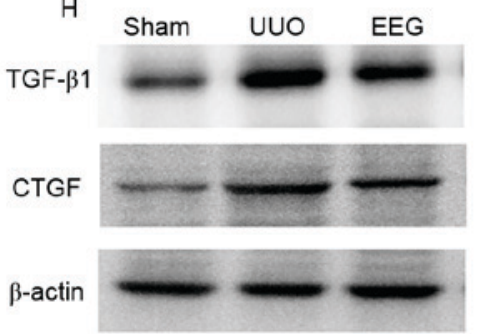

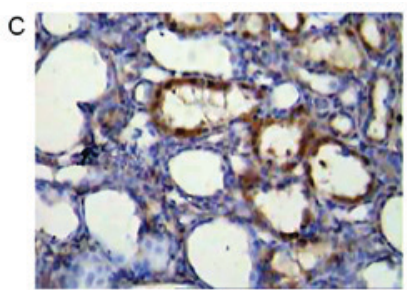
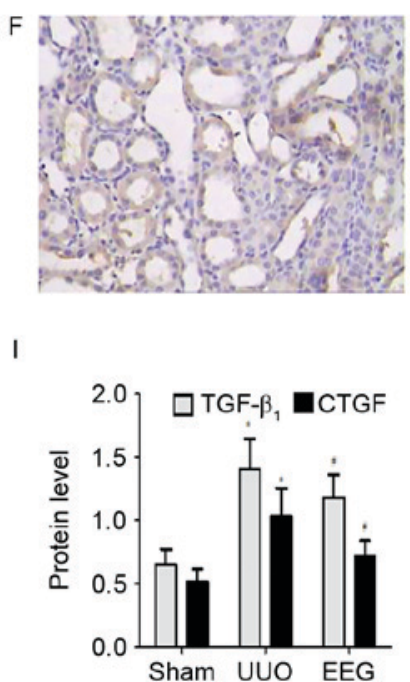

Figure 4. Immunostaining and western blot analysis of TGF- $\beta 1$ and CTGF. (A) TGF- $\beta 1$ staining was weak in the sham group. (B) TGF- $\beta 1$ staining was markedly increased in the UUO group. (C) TGF- $\beta 1$ expression was suppressed in the EEG group. (D) CTGF was weak in the sham group. (E) CTGF was markedly upregulated in the UUO group. (F) CTGF expression was suppressed in the EEG group. (G) The total IOD of TGF- $\beta 1$ and CTGF in each group. (H and I) Western blot analysis of TGF- $\beta 1$ and CTGF demonstrated similar trends as the immunohistochemistry findings. Images were captured at a magnification of $x 400$. Data are presented as the mean \pm standard deviation $(n=10)$. ${ }^{*} \mathrm{P}<0.05$ vs. sham group; ${ }^{*} \mathrm{P}<0.05$ vs. UUO group. TGF- $\beta 1$; transforming growth factor- $\beta 1$; CTGF, connective tissue growth factor; UUO, unilateral ureteral obstruction; EEG, ethanol extract of gardenia fruits; IOD, integrated optical density.

A

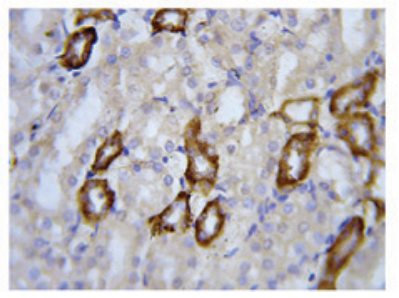

D

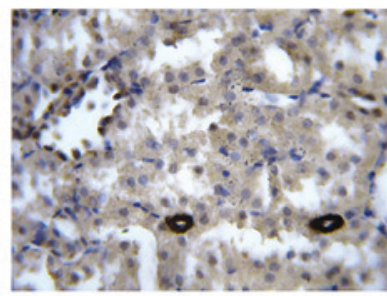

B
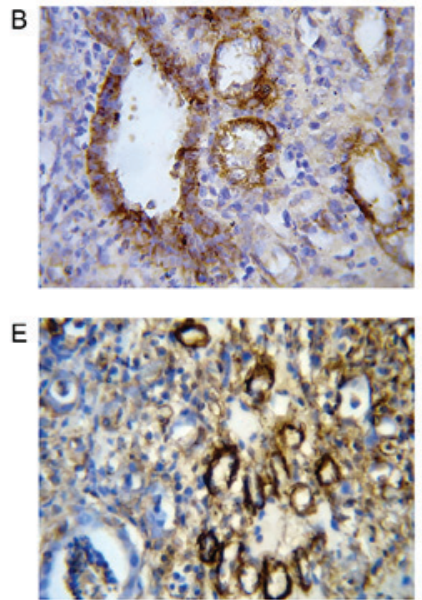
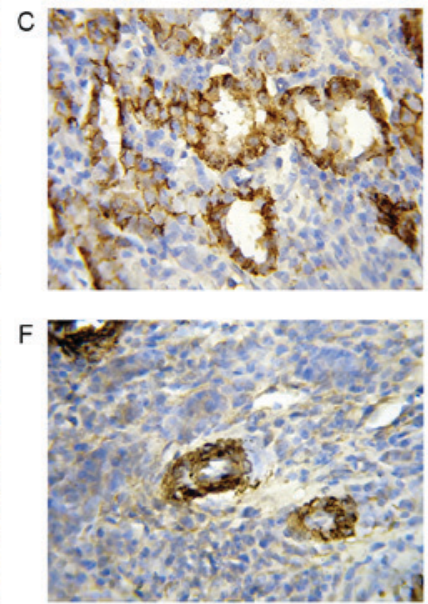

I
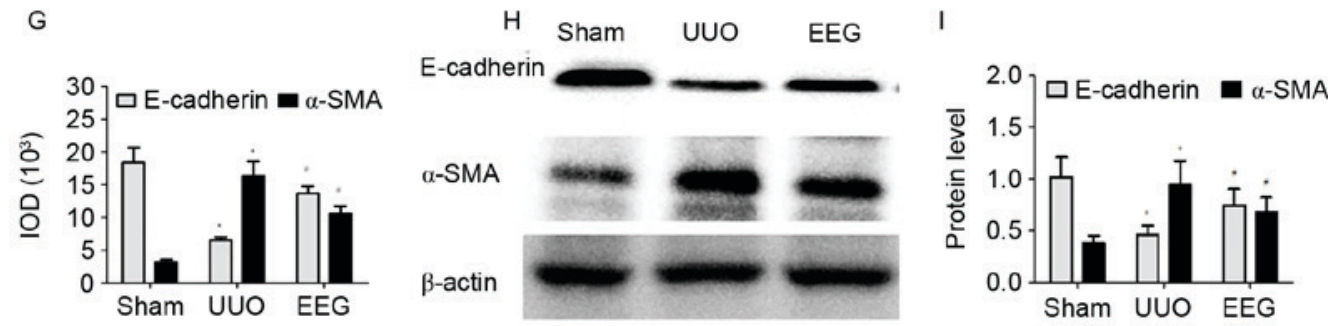

Figure 5. Immunostaining and western blot analysis of E-cadherin and $\alpha$-SMA. (A) E-cadherin staining was prominent in the sham group. (B) E-cadherin expression was weak in the UUO group. (C) E-cadherin expression was upregulated in the EEG group. (D) $\alpha$-SMA was weakly detected in the sham group. (E) $\alpha$-SMA was markedly increased in the UUO group. (F) $\alpha$-SMA expression was suppressed in the EEG group. (G) The total IOD of E-cadherin and $\alpha$-SMA in each group. (H and I) Western blot analysis of E-cadherin and $\alpha$-SMA expression demonstrated similar trends as the immunohistochemistry findings. Images were captured at a magnification of $\mathrm{x} 400$. Data are presented as the mean \pm standard deviation $(\mathrm{n}=10)$. ${ }^{*} \mathrm{P}<0.05$ vs. sham group; ${ }^{*} \mathrm{P}<0.05$ vs. $\mathrm{UUO}$ group. $\alpha$-SMA, $\alpha$-smooth muscle actin; UUO, unilateral ureteral obstruction; EEG, ethanol extract of gardenia fruits; IOD, integrated optical density. 
Fig. 5E and G). EEG treatment significantly reduced this $\alpha$-SMA expression ( $\mathrm{P}<0.05$; Fig. 5F and G). Similar observations were made in western blot analysis (Fig. $5 \mathrm{H}$ and I).

\section{Discussion}

Extraction with ethanol was used to obtain an extract of gardenia fruit that contained eight constituents including crocetin, and geniposide and genipin, which are marked antiinfective drugs. A recent study has discovered that crocetin treatment may protect against burn-induced small intestinal injury by inhibiting oxidative stress and inflammatory response (22). Furthermore, crocetin may alleviate cardiac, skin and lung fibrosis due to suppression of reactive oxygen species-dependent signaling pathways or a reduction in endothelin-1 $(23,24)$. It has also been reported that geniposide is able to suppress the release of pro-inflammatory cytokines tumor necrosis factor- $\alpha$, interleukin- 6 and interleukin- $1 \beta$ in vitro and in vivo, and is also able to block the phosphorylation of nuclear factor- $\mathrm{\kappa B}, \mathrm{p} 65$ and $\mathrm{p} 38$, as well as inhibiting the expression of extracellular signal-related kinases (ERKs) and c-Jun N-terminal kinases in lipopolysaccharide-stimulated primary mouse macrophages (16). Furthermore, geniposide is able to inhibit TGF- $\beta 1$-induced EMT in hepatic fibrosis by depressing the TGF- $\beta /$ Smad and ERK-mitogen-activated protein kinase signaling pathways (25).

Recent studies have demonstrated that EEG is protective against liver fibrosis $(19,26,27)$; however little focus has been given to the kidney. UUO rat is a mature experimental model for RIF in rats (22), which produces tubulointerstitial inflammation and fibrosis. In the present study, no impaired renal function was identified from BUN and Scr in UUO rats. These results were caused by an increase in unobstructed kidney functions, which compensated for the dysfunctions of obstructed-kidney. NAG in the UUO group was significantly raised when compared with the sham group, and EEG treatment significantly reduced this level.

In terms of pathology, the renal interstitium widened, and the tubules expanded in the UUO group. EEG was able to alleviate tubulointerstitial fibrosis induced by UUO. These results suggest that the UUO model for RIF was successfully established in the present study, and indicated that EEG is capable of depressing the progression of RIF.

It is widely accepted that EMT is an important mechanism associated with RIF (3). In EMT, expression of the epithelial cell marker E-cadherin and acquired mesenchymal features, such as expression of $\alpha$-SMA, are reduced in tubular epithelial cells. The present study demonstrated that EEG treatment significantly decreased $\alpha$-SMA and increased E-cadherin, indicating that EEG is able to inhibit the transition between epithelium and mesenchyme.

It has been demonstrated that TGF- $\beta 1$ is a profibrotic cytokine in the development of RIF, and it has been identified as the most potent inducer of EMT (8). CTGF is an important downstream mediator in the various profibrotic actions of TGF- $\beta 1$, which are associated with the degree of RIF and directly promote EMT $(9,28)$. In the present study, the level of CTGF and TGF- $\beta_{1}$ was significantly increased in the UUO group, whereas the expression of CTGF and TGF- $\beta_{1}$ in the EEG group were significantly decreased compared with the
UUO group. These results suggested that EEG decreased the expression of TGF- $\beta 1$ in UUO, and in turn reduced CTGF. Therefore, EEG may inhibit EMT induction by UUO via TGF- $\beta 1$ and CTGF.

Hypoxia has been recognized as a crucial microenvironmental factor in the progression of tissue fibrosis (29). Under hypoxic condition, HIF-1 $\alpha$ dimerizes with HIF-1 $\beta$ and this heterodimer HIF-1 translocates to the nucleus, where the activation of target genes is mediated by binding to hypoxia-response elements (30). HIF- $1 \alpha$ is a sensitive hypoxia indicator, which may be a crucial factor in EMT $(31,32)$. HIF-1 $\alpha$ in the UUO group was significantly higher than that in the sham group, and following EEG treatment, the expression of HIF-1 $\alpha$ was significantly lower than that of the UUO group, suggesting that hypoxia was ameliorated in the EEG group. A previous study suggested that hypoxia and signaling through HIF-1 $\alpha$ contribute to the development of interstitial fibrosis via the induction of ECM-modifying and the modulation of EMT (32). The present results indicate that EEG inhibited EMT through HIF- $1 \alpha$ in addition to TGF- $\beta 1$ and CTGF.

VEGF is a survival factor for vascular endothelial cells and serves an important role in the homeostasis of the vascular endothelium, which is induced by hypoxia and transcriptionally activated by HIF-1 (33). A reduction of VEGF was observed in the UUO group. The decrease of VEGF was coincident with severe interstitial fibrosis. This data may be contrary to the result of elevated HIF-1 $\alpha$ in this model. Several studies have suggested that transcription factor Sp1 and the oncogene Ras are transcriptional activators of VEGF $(34,35)$. Consequently, the reduction of VEGF levels in UUO may be the result of multiple factors in addition to hypoxia and HIF-1 $\alpha$ expression. The level of VEGF in the EEG group was significantly increased compared with the UUO group. It was previously reported that VEGF enhanced glomerular capillary repair and prevented the progression of kidney diseases (36). VEGF is greatly expressed in tubular epithelial cells, and renal tubular epithelial cells are an important cellular resource of VEGF. There may be an intrinsic renoprotective reason that maintains the epithelial phenotype of tubular cells, and loss of these factors may contribute to EMT progression. VEGF may be a candidate for this type of factor as reduced expression of VEGF has been observed in RIF $(37,38)$. Furthermore, it has been demonstrated previously that VEGF inhibited EMT through its influence on TGF- $\beta 1$ and CTGF in UUO mice (39). Therefore, it may be speculated that EEG treatment decreased the expressions of HIF- $1 \alpha$ and increased VEGF, inhibiting fibrosis and EMT through decreasing TGF- $\beta 1$ and CTGF.

In conclusion, the present study demonstrated that EEG had a protective effect on UUO rats, and may be able to alleviate renal fibrosis and regulate EMT via decreasing the expression of HIF- $1 \alpha$, CTGF, and TGF- $\beta_{1}$, and elevating VEGF protein levels. These results provide rationale for the further study of the mechanisms of EEG and identify a potential novel therapeutic method for the treatment of RIF.

\section{Acknowledgments}

The present study was supported by the Joint Fund of Science and Technology Department of Guizhou Province [grant 
no. J(2010)2], the Project Natural Science Foundation of China (grant no. 21562051) and the project of Science and Technology Agency of Guizhou Province [grant no. J(2015) 2157].

\section{References}

1. Jha V, Garcia-Garcia G, Iseki K, Li Z, Naicker S, Plattner B, Saran R, Wang AY and Yang CW: Chronic kidney disease: Global dimension and perspectives. Lancet 382: 260-272, 2013.

2. Lozano R, Naghavi M, Foreman K, Lim S, Shibuya K, Aboyans V, Abraham J, Adair T, Aggarwal R, Ahn SY, et al: Global and regional mortality from 235 causes of death for 20 age groups in 1990 and 2010: A systematic analysis for the global burden of disease study 2010. Lancet 380: 2095-2128, 2012.

3. Sun YB, Qu X, Caruana G and Li J: The origin of renal fibroblasts/myofibroblasts and the signals that trigger fibrosis. Differentiation 92: 102-107, 2016.

4. Duffield JS, Lupher M, Thannickal VJ and Wynn TA: Host responses in tissue repair and fibrosis. Annu Rev Pathol 8 : 241-276, 2013

5. Bertinat R, Silva P, Mann E, Li X, Nualart F and Yáñez AJ: In vivo sodium tungstate treatment prevents $\mathrm{E}$-cadherin loss induced by diabetic serum in HK-2 cell line. J Cell Physiol 230: 2437-2446, 2015.

6. Wang JY, Gao YB, Zhang N, Zou DW, Wang P, Zhu ZY, Li JY, Zhou SN, Wang SC, Wang YY and Yang JK: miR-21 overexpression enhances TGF- $\beta 1$-induced epithelial-to-mesenchymal transition by target smad7 and aggravates renal damage in diabetic nephropathy. Mol Cell Endocrinol 392: 163-172, 2014.

7. Farris $\mathrm{AB}$ and Colvin RB: Renal interstitial fibrosis: Mechanisms and evaluation. Curr Opin Nephrol Hypertens 21: 289-300, 2012.

8. López-Hernández FJ and López-Novoa JM: Role of TGF- $\beta$ in chronic kidney disease: An integration of tubular, glomerular and vascular effects. Cell Tissue Res 347: 141-154, 2012.

9. Phanish MK, Winn SK and Dockrell ME: Connective tissue growth factor-(CTGF, CCN2)-a marker, mediator and therapeutic target for renal fibrosis. Nephron Exp Nephrol 114: e83-e92, 2010.

10. Cammarata PR, Neelam S and Brooks MM: Inhibition of hypoxia inducible factor-1 $\alpha$ downregulates the expression of epithelial to mesenchymal transition early marker proteins without undermining cell survival in hypoxic lens epithelial cells. Mol Vis 21: 1024-1035, 2015.

11. Sun Y, Wang H, Liu M, Lin F and Hua J: Resveratrol abrogates the effects of hypoxia on cell proliferation, invasion and EMT in osteosarcoma cells through downregulation of the HIF-1 $\alpha$ protein. Mol Med Rep 11: 1975-1981, 2015.

12. Hakroush S, Moeller MJ, Theilig F, Kaissling B, Sijmonsma TP, Jugold M, Akeson AL, Traykova-Brauch M, Hosser H, Hähnel B, et al: Effects of increased renal tubular vascular endothelial growth factor (VEGF) on fibrosis, cyst formation, and glomerular disease. Am J Pathol 175: 1883-1895, 2009.

13. Sis B, Husain S, Chang J, Halloran P and Osasan S: Decreased renal VEGF-A signaling as a mechanism for kidney transplant fibrosis and failure. Am J Transplant 13 (Suppl 5): S110, 2013.

14. Wang YR, Chen Y, Deng L, Cai S, Liu J, Li W, Du L, Cui G, $\mathrm{Xu}$ X, Lu T, et al: Systematic separation and purification of iridoid glycosides and crocetin derivatives from Gardenia jasminoides Ellis by high-speed counter-current chromatography. Phytochem Anal 26: 202-208, 2015.

15. Hong YJ and Yang KS: Anti-inflammatory activities of crocetin derivatives from processed Gardenia jasminoides. Arch Pharm Res 36: 933-940, 2013.

16. Fu YH, Liu B, Liu J, Liu Z, Liang D, Li F, Li D, Cao Y, Zhang X, Zhang N and Yang Z: Geniposide, from Gardenia jasminoides Ellis, inhibits the inflammatory response in the primary mouse macrophages and mouse models. Int Immunopharmacol 14: 792-798, 2012

17. Koo HJ, Lim KH, Jung HJ and Park EH: Anti-inflammatory evaluation of gardenia extract, geniposide and genipin. J Ethnopharmacol 103: 496-500, 2006.

18. Chang KH, Chen WL, Wu YR, Lin TH, Wu YC, Chao CY, Lin JY, Lee LC, Chen YC, Lee-Chen GJ and Chen CM: Aqueous extract of Gardenia jasminoides targeting oxidative stress to reduce polyQ aggregation in cell models of spinocerebellar ataxia 3. Neuropharmacology 81: 166-175, 2014.
19. Chen YH, Lan T, Li J, Qiu CH, Wu T, Gou HJ and Lu MQ Gardenia jasminoides attenuates hepatocellular injury and fibrosis in bile duct-ligated rats and human hepatic stellate cells. World J Gastroenterol 18: 7158-7165, 2012.

20. Mizuno S, Matsumoto K and Nakamura T: Hepatocyte growth factor suppresses interstitial fibrosis in a mouse model of obstructive nephropathy. Kidney Int 59: 1304-1314, 2001.

21. Mizuguchi Y, Miyajima A, Kosaka T, Asano T, Asano T and Hayakawa M: Atorvastatin ameliorates renal tissue damage in unilateral ureteral obstruction. J Urol 172: 2456-2459, 2004.

22. Zhou C, Bai W, Chen Q, Xu Z, Zhu X, Wen A and Yang X Protective effect of crocetin against burn-induced intestinal injury. J Surg Res 198: 99-107, 2015.

23. Song Y, Zhu L and Li M: Antifibrotic effects of crocetin in scleroderma fibroblasts and in bleomycin-induced sclerotic mice. Clinics (Sao Paulo) 68: 1350-1357, 2013.

24. Cai J, Yi FF, Bian ZY, Shen DF, Yang L, Yan L, Tang QZ, Yang $\mathrm{XC}$ and $\mathrm{Li} \mathrm{H}$ : Crocetin protects against cardiac hypertrophy by blocking MEK-ERK1/2 signalling pathway. J Cell Mol Med 13: 909-925, 2009

25. Park JH, Yoon J, Lee KY and Park B: Effects of geniposide on hepatocytes undergoing epithelial-mesenchymal transition in hepatic fibrosis by targeting TGF $\beta /$ Smad and ERK-MAPK signaling pathways. Biochimie 113: 26-34, 2015.

26. Chen P, Chen Y, Wang Y, Cai S, Deng L, Liu J and Zhang H: Comparative evaluation of hepatoprotective activities of geniposide, crocins and crocetin by CCl4-induced liver injury in mice. Biomol Ther (Seoul) 24: 156-162, 2016.

27. Ma T, Huang C, Zong G, Zha D, Meng X, Li J and Tang W: Hepatoprotective effects of geniposide in a rat model of nonalcoholic steatohepatitis. J Pharm Pharmacol 63: 587-593, 2011.

28. Sanz-Rosa D, de las Heras N, Ortega MR, et al: Role of CTGF on renal fibrosis in spontaneously hypertensive rats. Effect of treatment with candesartan. J Hypertens 23: 327-327, 2005.

29. Haase VH: Pathophysiological consequences of HIF activation: HIF as a modulator of fibrosis. Ann N Y Acad Sci 1177: 57-65, 2009.

30. Nørregaard R, Bødker T, Jensen BL, Stødkilde L, Nielsen S and Frøkiaer J: Increased renal adrenomedullin expression in rats with ureteral obstruction. Am J Physiol Regul Integr Comp Physiol 296: R185-R192, 2009.

31. Harris AL: Hypoxia-a key regulatory factor in tumour growth. Nat Rev Cancer 2: 38-47, 2002.

32. Higgins DF, Kimura K, Bernhardt WM, Shrimanker N, Akai Y, Hohenstein B, Saito Y, Johnson RS, Kretzler M, Cohen CD, et al: Hypoxia promotes fibrogenesis in vivo via HIF-1 stimulation of epithelial-to-mesenchymal transition. J Clin Invest 117: 3810-3820, 2007.

33. Hellwig-Bürgel T, Stiehl DP, Katschinski DM, Marxsen J, Kreft B and Jelkmann W: VEGF production by primary human renal proximal tubular cells: Requirement of HIF-1, PI3-kinase and MAPKK-1 signaling. Cell Physiol Biochem 15: 99-108, 2005.

34. Taoka R, Jinesh GG, Xue W, Safe S and Kamat AM: CF3DODA-Me induces apoptosis, degrades $\mathrm{Sp1}$, and blocks the transformation phase of the blebbishield emergency program. Apoptosis 22: 719-729, 2017.

35. Milanini-Mongiat J, Pouysségur $\mathrm{J}$ and Pagès G: Identification of two Sp1 phosphorylation sites for p42/p44 mitogen-activated protein kinases: Their implication in vascular endothelial growth factor gene transcription. J Biol Chem 277: 20631-20639, 2002.

36. Wahab NA and Mason RM: A critical look at growth factors and epithelial-to-mesenchymal transition in the adult kidney. Interrelationships between growth factors that regulate EMT in the adult kidney. Nephron Exp Nephrol 104: 129-134, 2006.

37. Song YR, You SJ, Lee YM, Chin HJ, Chae DW, Oh YK, Joo KW, Han JS and Na KY: Activation of hypoxia-inducible factor attenuates renal injury in rat remnant kidney. Nephrol Dial Transplant 25: 77-85, 2010.

38. Burt LE, Forbes MS, Thornhill BA, Kiley SC and Chevalier RL: Renal vascular endothelial growth factor in neonatal obstructive nephropathy. I. Endogenous VEGF. Am J Physiol Renal Physiol 292: F158-F167, 2007.

39. Lian YG, Zhou QG, Zhang YJ and Zheng FL: VEGF ameliorates tubulointerstitial fibrosis in unilateral ureteral obstruction mice via inhibition of epithelial-mesenchymal transition. Acta Pharmacol Sin 32: 1513-1521, 2011. 